\title{
Psychologische Sicherheit als Voraussetzung für Innovativität und Flexibilität in Teams und Organisationen
}

\author{
Thomas Bachmann • Heidi Möller
}

Angenommen: 6. Juli 2021 / Online publiziert: 9. August 2021

(C) Der/die Autor(en) 2021

Mit der Idee von New Work (Bergmann 2019) und den damit zunehmenden experimentellen Veränderungen in der Arbeitswelt hinsichtlich Organisationsstrukturen, Führung und Zusammenarbeit ergeben sich viele Möglichkeiten und Perspektiven für die beteiligten Menschen: Sinnhaftigkeit, Flexibilität, Ganzheitlichkeit und Kreativität bekommen mehr Raum und kommen Teams und Organisationen im Wettbewerb um die besten Köpfe und die besten Ideen zugute (Schermuly 2016). Gleichzeitig jedoch verlangen die neuen Formen der Zusammenarbeit auch ganz neue persönliche Kompetenzen, wie der Umgang mit Unsicherheit und Vorläufigkeit, intensives Kooperieren und Unterstützen von anderen oder etwa Feedbackfähigkeit und zunehmend mehr Resilienz. Dies betrifft zunächst jeden Einzelnen, in letzter Konsequenz jedoch vor allem die sozialen Systeme, wie Gruppen, Teams und Organisationen deren Zusammenspiel determinieren, inwieweit die Vorteile von New Work in der Teamarbeit und auf Organisationsebene zum Tragen kommen können.

Mit dem Konzept der psychologischen Sicherheit wurde schon sehr früh begründet und in zahlreichen Studien bestätigt, was die Voraussetzungen und Erfolgsfaktoren für Veränderungsfähigkeit, Innovativität und Flexibilität von Menschen und damit auch von sozialen Systemen sind (Edmondson und Lei 2014; Schein und Bennis 1965). Psychologische Sicherheit bedeutet für Menschen in Teams und Organisationen, gefahrlos interpersonelle Risiken eingehen zu können, das heißt, ein Verhalten zu zeigen, das oftmals unterdrückt, abgewertet oder lächerlich gemacht wird.

PD Dr. Thomas Bachmann $(\bowtie)$

Institut für Psychologie, Humboldt-Universität zu Berlin, Unter den Linden 6, 10099 Berlin,

Deutschland

E-Mail: thomas.bachmann@hu-berlin.de

Prof. Dr. Heidi Möller

Institut für Psychologie, Universität Kassel, Holländische Straße 36-38, 34127 Kassel, Deutschland

E-Mail: heidi.moeller@uni-kassel.de 
Ein psychologisch sicheres Team würde diese Verhaltensweise nicht nur tolerieren, sondern sie sogar befördern: Kritik üben, abweichende Meinungen äußern, ungewöhnliche Ideen einbringen, andere um Unterstützung bitten sind wichtige Verhaltensweisen, die die Zusammenarbeit, Kreativität und Innovativität in Arbeitsteams fördern. Mit dem Themenschwerpunkt dieses Heftes wollen wir mit den wichtigsten Aspekten psychologischer Sicherheit vertraut machen sowie deren Bedeutsamkeit in unterschiedlichen Kontexten beleuchten.

Im Beitrag von Erin Burell und Elisabeth Brauner wird das Zusammenspiel von psychologischer Sicherheit und einem funktionierenden transactive memory system in Gruppen und Teams näher beleuchtet. Dessen Entstehen ist ein wichtiges Merkmal erfolgreicher Teamarbeit. Nur durch das Wissen, wer im Team über welches Wissen und welche Fähigkeiten verfügt, kann ein Team das werden, was es ist, nämlich mehr als die Summe seiner Mitglieder. Die Autorinnen arbeiten sehr schön heraus, dass sich psychologische Sicherheit und das transactive memory system wechselseitig hervorbringen und bedingen und daher nicht losgelöst voneinander zu betrachten sind.

Der Beitrag von Thomas Bachmann und Katherina Quispe Bravo führt diese Gedanken auf Teamebene fort und stellt die Frage, welche Teamfaktoren das Entstehen psychologischer Sicherheit im Team bedingen und inwieweit psychologische Sicherheit mit der Identifikation mit dem Team und seiner Aufgabe in Zusammenhang steht. Die Ergebnisse verweisen auf interessante Zusammenhänge zwischen psychologischer Sicherheit und Teamidentifikation gerade im Zusammenhang mit der Corona-Krise, indem sie zeigen, wie wichtig die persönliche Begegnung für die Identifikation mit der eigenen Arbeitsgruppe ist.

In dem Artikel von Alexander Pfurr und Freimut Schirrmacher wird ein Aspekt in Zusammenhang mit psychologischer Sicherheit beleuchtet, der bisher in der Literatur kaum Beachtung fand, nämlich wie wichtig psychologische Sicherheit nach Übernahmen oder Fusionen ist, um die Integration neuer Mitarbeitenden zu ermöglichen bzw. zu erleichtern. Denn die Reibungsverluste hier machen oftmals die durch Unternehmensfusionen erhofften Synergien wieder zunichte, wenn sich Widerstände und Absentismus ausbreiten. Die Autoren bieten dafür ein sinnfälliges Modell an, das für die Gestaltung solcher Prozesse handlungsleitend sein kann.

Birgit Feldhusen widmet sich in ihrem Beitrag dem Zusammenhang zwischen kollektiver Intelligenz und psychologischer Sicherheit und kann darin herleiten, dass kollektive Intelligenz nicht nur ein rationales Phänomen der Addition von Wissen und Fähigkeiten, sondern vor allem ein soziales Phänomen ist. Auch hier kann psychologische Sicherheit ein hilfreiches Konstrukt sein, weil es erklärt, welche Qualitäten des sozialen Miteinanders im Team von Bedeutung sind, damit es zu einer Beziehungs- und Begegnungsqualität kommt, die Wissensaustausch, das Teilen von Erfahrungen und die wechselseitige Unterstützung fördert.

Unabhängig von diesem Themenschwerpunkt folgen einige weitere Beträge: Anna-Lorena De Luca geht in ihrer empirischen Untersuchung den Geheimnissen in der Supervision nach. Am Beispiel der Supervision für Psychotherapeut/innen in Ausbildung (PiAs) zeigt sie auf, welche Tabuthemen es in deren Supervision gibt und was die Motive der Supervisand/innen dafür sind, Dinge zu verschweigen, zu beschönigen oder zu erfinden. Supervision als Ort der professionellen Selbstreflexion 
setzt eine gute Arbeitsbeziehung und vor allem Aufrichtigkeit voraus. In erschreckender Weise kann sie zeigen, dass viele Kandidat/innen sich nicht getrauen, sich zu äußern, wenn sie das Format als wenig hilfreich erleben, sich nicht ernst genommen fühlen, wenn sie in ihren psychotherapeutischen Konzeptionen von denen der Anleiter/innen abweichen, aggressive Gegenübertragungsgefühle der Supervisorin gegenüber empfinden, die Schweigepflicht verletzen oder sich mit dem Zweifel am professionellen Selbstwert herumschlagen. De Lucas Arbeit fordert dazu auf, in anderen Feldern der Supervision repliziert zu werden. Denn wie könnten wir annehmen, dass sich die Lage in unseren Arbeitsfeldern anders darstellen würde?

Der Lehrstuhl Theorie und Methodik der Beratung der Universität Kassel verfolgt seit langem wissenschaftlich die Kompetenzentwicklung von Menschen, die in der Beratung bzw. Psychotherapie arbeiten. In diesem Beitrag von Alessa Antonia Müller und Heidi Möller wird ein aus der Psychotherapieforschung an die Coachingwirklichkeit angepasstes Instrument vorgelegt, das die interpersonellen Fähigkeiten, über die Coaches verfügen sollten, in einem Prä-Post-Vergleich abbilden kann. Postgraduale Studierende führten zu Beginn ihrer Ausbildung erste audiographierte Coachinggespräche, und das Gleiche taten sie nach einem Jahr des Studiums. Die Autorinnen stellen in ihrer explorativen Studie ein Instrument vor, das die professionelle Entwicklung zum Coach jenseits subjektiver Rückmeldungen der Lehrenden auf objektivierte Füße stellt.

Im folgenden Praxisbericht präsentieren Christine Andres Roduit und MarieChristine Walter ihr „GUIDE-Modell“: Karriereentscheidungen sind in deutlich länger werdenden Arbeitsbiographien an der Tagesordnung. Will ich die Stelle wechseln? Will ich den hierarchischen Aufstieg? Stelle ich mich neuen beruflichen Herausforderungen? Welche Stelle passt zu mir und welche Weiterbildung macht für meine Entwicklung Sinn? Das GUIDE-Modell stellt ein strukturiertes Vorgehen bereit, das Beratenden und ihrer Klientel ermöglicht, ihre Laufbahn zu gestalten. Ausgehend von einer Begriffsbestimmung Laufbahnberatung wird das ressourcenstärkende Modell des Gap, Understanding and Improving, Developing solutions, Execution (GUIDE) in seinen Elementen erläutert: Wissen und Kompetenzen, die Frage der Identität, aber auch situative Umfeldfaktoren sowie Motivation greifen wie Zahnräder ineinander.

Schließlich nimmt uns Thomas Bachmann noch auf ein kleines systemisches Gedankenexperiment in einer Kulturanalyse mit. Der Film „Und täglich grüßt das Murmeltier“ lädt gerade dazu ein, sich mit doppelter Kontingenz, Abschlusskommunikation, Musterbildung und Selbstreferenz zu beschäftigen, um zu erfahren, warum es nicht viel braucht, um mit anderen im Dialog persönlich zu wachsen.

In der Rubrik „Diskurs“ wollen wir nach der Abfolge von Beiträgen zur CoronaKrise eine weitere Diskussionsrunde eröffnen: Mit der Veröffentlichung von Böning und Strikker (2020) „Coaching in der zweiten Romantik“ wurde eine Diskussion angestoßen, auf die die hier folgenden Essays von Uwe Böning, Heidi Möller und Thomas Giernalczyk Bezug nehmen und Stellung beziehen zur „Neuorientierung für das Business-Coaching - Anfragen zur gesellschaftlichen Verantwortung von Coaches“. Im Kern geht es dabei um eine Betrachtung und Analyse der Entwicklung des Coachings, vor allem des Business-Coachings, das nicht vor einigen starken und gegensätzlichen Herausforderungen steht, sondern sich schon mittendrin befindet. 
Man kann das sehr rational und sehr emotional betrachten - je nach Temperament und Interesse. Auf alle Fälle sind die anstehenden Entwicklungen und die damit verbundenen Fragen nicht nur spannend, sie dürften vielmehr schnell zu zentralen Themen werden, die das Coaching aus verschiedenen Richtungen betrachten: als methodengeleitete Praxis, als Theorie und Wissenschaft und als Wirtschaftsbranche. Als ikonografische Figur im Schnittpunkt verschiedenster externer gesellschaftlicher und politischer wie auch fachinterner Entwicklungen wird Coaching somit massiven Veränderungskräften ausgesetzt, die zur Reflexion der Lage zwingen. Sie werden deshalb auch zu Positionen drängen, die die Zukunft des Coachings fundamental betreffen. $\mathrm{Zu}$ umfassend und gegenläufig, zusammenhängend wie widersprüchlich oder fast unabhängig sind die Themen und Entwicklungen, als dass sie nur still und ergeben hingenommen werden dürften.

Die Autor/innen stellen sich dieser Diskussion und fordern zur weiteren offenen Diskussion auf, damit die Chancen des Coachings für die Zukunft ausgeleuchtet, aber auch seine Grenzen wahrnehmbar werden. Diesem ersten Teil der Veröffentlichung widmen sich die Autor/innen in der Hoffnung, dass weitere Aspekte im Laufe der Auseinandersetzung in den Fokus kommen werden. Bitte beteiligen Sie sich, lieber Leserinnen und Leser, daran!

Viel Freude beim Weiterdenken wünschen

Heidi Möller und Thomas Bachmann

Funding Open Access funding enabled and organized by Projekt DEAL.

Open Access Dieser Artikel wird unter der Creative Commons Namensnennung 4.0 International Lizenz veröffentlicht, welche die Nutzung, Vervielfältigung, Bearbeitung, Verbreitung und Wiedergabe in jeglichem Medium und Format erlaubt, sofern Sie den/die ursprünglichen Autor(en) und die Quelle ordnungsgemäß nennen, einen Link zur Creative Commons Lizenz beifügen und angeben, ob Änderungen vorgenommen wurden.

Die in diesem Artikel enthaltenen Bilder und sonstiges Drittmaterial unterliegen ebenfalls der genannten Creative Commons Lizenz, sofern sich aus der Abbildungslegende nichts anderes ergibt. Sofern das betreffende Material nicht unter der genannten Creative Commons Lizenz steht und die betreffende Handlung nicht nach gesetzlichen Vorschriften erlaubt ist, ist für die oben aufgeführten Weiterverwendungen des Materials die Einwilligung des jeweiligen Rechteinhabers einzuholen.

Weitere Details zur Lizenz entnehmen Sie bitte der Lizenzinformation auf http://creativecommons.org/ licenses/by/4.0/deed.de.

\section{Literatur}

Bergmann, F. (2019). New work new culture: work we want and a culture that strengthens us. Hampshire: John Hunt Publishing.

Böning, U., \& Strikker, F. (2020). Coaching in der zweiten Romantik: Abstieg oder Aufstieg? Zwischen individuellem Glücksversprechen und gesellschaftlicher Verantwortung. Wiesbaden: Springer. essentials

Edmondson, A. C., \& Lei, Z. (2014). Psychological safety: the history, renaissance, and future of an interpersonal construct. Annu. Rev. Organ. Psychol. Organ. Behav., 1(1), 23-43.

Schein, E.H., \& Bennis, W. G. (1965). Personal and organizational change through group methods: the laboratory approach. New York: Wiley.

Schermuly, C.C. (2016). New Work - Gute Arbeit gestalten: Psychologisches Empowerment von Mitarbeitern. Bd. 10167. Freiburg: Haufe-Lexware. 